\title{
MULTI-BAND OFDM: A NEW APPROACH FOR UWB
}

\author{
Anuj Batra, Jaiganesh Balakrishnan, and Anand Dabak \\ DSPS R\&D Center, Texas Instruments, 12500 TI Blvd, MS 8649, Dallas, TX 75206 \\ \{batra,jai, dabak\}@ti.com
}

\begin{abstract}
In this paper, a multi-band OFDM (MB-OFDM) system for ultra-wideband (UWB) communication is described. In this system, the UWB spectrum is divided into bands that are $528 \mathrm{MHz}$ wide and the data is transmitted across the bands using a time-frequency code. Within each subband, an OFDM modulation scheme is used to transmit the information. An overview of the MB-OFDM system and various systems design considerations are discussed. In addition, the performance results in realistic multi-path environments are provided for the MB-OFDM system.
\end{abstract}

\section{INTRODUCTION}

Over the last year and half, UWB communication systems have received significant attention from both the industry and as well as academia. The primary reason for the increased attention is due to the landmark ruling by the Federal Communications Commission (FCC). In February 2002, the FCC allocated 7,500 MHz of spectrum (from $3.1 \mathrm{GHz}$ to $10.6 \mathrm{GHz}$ ) for use by UWB devices. This ruling has helped to create new standardization efforts, like IEEE 802.15.3a, that focus on developing high-speed wireless communication systems for personal area network.

Another reason for the excitement about UWB is because this technology promises to deliver data rates that can scale from a data rate of $110 \mathrm{Mb} / \mathrm{s}$ at a distance of 10 meters up to a data rate of $480 \mathrm{Mb} / \mathrm{s}$ at a distance of 2 meters in realistic multi-path environments all while consuming very little power and silicon area. It is expected that UWB devices will be able to provide low cost solutions that can satisfy the consumers need for data rates as well as enable new consumer market segments.

The paper is organized as follows. Section 2 provides a description of various UWB approaches and motivates the design of a MB-OFDM system. Section 3 describes the system parameters for the MB-OFDM system. Some of the design considerations for a MB-OFDM solution are discussed in section 4 . Section 5 provides performance results of the MB-OFDM system based on detailed link level simulations and section 6 concludes.

\section{OVERVIEW OF PREVIOUS UWB SYSTEMS}

The traditional design approach for UWB communication system involved using narrow time-domain pulses that occupy a very wide spectrum [1]. The main disadvantage of these systems is the challenge of building RF and analog circuits with large bandwidths, high speed analogto-digital converters (ADCs) to process this extremely wideband signal, and the significant digital complexity required to capture the multi-path energy in dense multipath environments.

An approach based on pulsed multi-band [2] alleviated the need to process the signal over a large bandwidth. This approach divides the spectrum into several smaller subbands, whose bandwidth is approximately $500 \mathrm{MHz}$, and single-carrier modulation techniques are used to transmit the information on each of the sub-bands. By interleaving the symbols across sub-bands, the UWB system can still maintain the same transmit power as if it were using the entire bandwidth. By using smaller sub-bands to transmit the information, the processing bandwidth for the receiver can be reduced, thereby lowering the complexity of the design, reducing the power consumption of the radio, lowering the overall solution cost, and improving spectral flexibility and worldwide compliance.

The primary disadvantage of a pulsed multi-band system is the difficulty in collecting significant multi-path energy when using a single RF chain. The amount of multi-path energy collected is limited by the dwell time on each subband. In addition, when a small number of RAKE fingers are employed, the system performance is very sensitive to group delay variations introduced by the analog front-end components. Finally, the pulsed multi-band system places very stringent band-switching timing requirements $(<100$ ps) at both the transmitter and receiver, leading to complicated synthesizer designs.

Multi-path energy collection is an important issue because it fundamentally determines the range and robustness of a communications system. By combining orthogonal frequency division multiplexing (OFDM) system with multi-banding, the strengths of the pulsed multi-band system can be retained while still addressing the issue of 
multi-path energy capture. This new system, MB-OFDM $[3,4]$, has several nice properties, including the ability to efficiently capture multi-path energy with a single RF chain, insensitivity to group delay variations, and the ability to deal with narrowband interferers at the receiver without having to sacrifice either sub-bands or data rate. In addition, the MB-OFDM system offers simplified synthesizer architectures and relaxes the band-switching timing requirements. The only potential drawback of this MB-OFDM approach is that the transmitter is more complex because of the IFFT and slightly higher peak-toaverage ratio than a pulse-based multi-band system.

\section{SYSTEM OVERVIEW OF MULTI-BAND OFDM}

For the sake of simplicity, a MB-OFDM system consisting only three bands is described herein. Figure 1 illustrates how the OFDM symbols are transmitted in a MB-OFDM system. In this example, it has been implicitly assumed that the time-frequency coding (TFC) is performed across just three OFDM symbols; however, in practice, the TFC pattern can have a much longer periodicity.

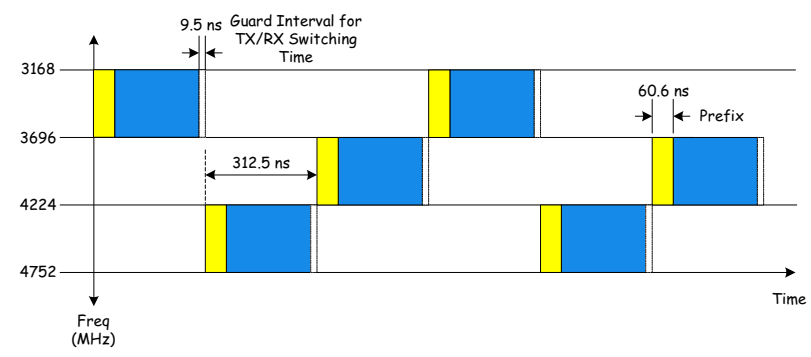

Figure 1 - Example of TF coding for an MB-OFDM system.

In a MB-OFDM system, a guard interval (9.5 nanoseconds) is appended to each OFDM symbol and a zero-padded prefix (60.6 nanoseconds) is inserted at the beginning of each OFDM symbol. The guard interval ensures that there is sufficient time for the transmitter and receiver to switch to the next carrier frequency. A zeropadded prefix provides both robustness against multi-path and eliminates the need for power back-off at the transmitter. More details about the zero-padded prefix will be described in a later section.

An example of a block diagram for a transmitter architecture that implements the MB-OFDM system is shown in Figure 2. The structure of the MB-OFDM solution is very similar to that of a conventional wireless OFDM physical layer, except that the carrier frequency is changed based on the time-frequency code. In addition, other modifications have been made to reduce the complexity. For example, the constellation size has been limited to QPSK in order to reduce the internal precision of the digital logic, specifically the IFFT and FFT, and to reduce the required precision of the ADC and DAC. The larger spacing between the sub-carriers, as compared to narrow-band OFDM systems such as IEEE 802.11a, also contributes to the simplicity of the MB-OFDM implementation. The large spacing relaxes the phase noise requirements on the carrier synthesis circuitry and improves robustness to synchronization errors.

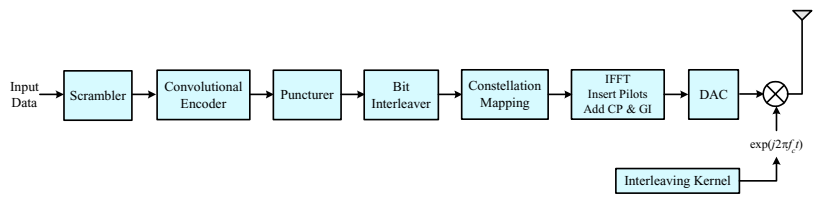

Figure 2 - Example architecture for a MB-OFDM system.

\subsection{System Parameters}

The system parameters for the $110 \mathrm{Mb} / \mathrm{s}, 200 \mathrm{Mb} / \mathrm{s}$, and $480 \mathrm{Mb} / \mathrm{s}$ modes of MB-OFDM solution are given in Table 1. Note that this system is also capable of transmitting additional data rates of 55, 80, 160, and 320 $\mathrm{Mb} / \mathrm{s}$. This system employs an orthogonal frequency division multiplexing modulation schemes with a total of 128 sub-carriers, only 122 of which carry energy. Of the 122 sub-carriers that carry energy, 100 are devoted to data, 12 are assigned to pilot tones, and the remaining 10 are used as guard tones.

Table 1 - MB-OFDM System Parameters

\begin{tabular}{|l|l|l|l|}
\hline Data Rate & $110 \mathrm{Mb} / \mathrm{s}$ & $200 \mathrm{Mb} / \mathrm{s}$ & $480 \mathrm{Mb} / \mathrm{s}$ \\
\hline Constellation & QPSK & QPSK & QPSK \\
\hline FFT Size & 128 & 128 & 128 \\
\hline Coding Rate & $11 / 32$ & $5 / 8$ & $3 / 4$ \\
\hline F-D Spreading & No & No & No \\
\hline T-D Spreading & Yes & Yes & No \\
\hline Symbol Length & $312.5 \mathrm{~ns}$ & $312.5 \mathrm{~ns}$ & $312.5 \mathrm{~ns}$ \\
\hline
\end{tabular}

Forward error correction coding in conjunction with either frequency-domain spreading or time-domain spreading is used to vary the data rate of the system. The different coding rates are generated by puncturing an industry standard $\mathrm{R}=1 / 3, \mathrm{~K}=7$ convolutional code with generator polynomial $[133,145,175]_{8}$. The exact puncturing patterns to generate the various coding rates are specified in [3]. The frequency-domain spreading is obtained by forcing the input data into the IFFT to be conjugate symmetric. An advantage of using this frequency-domain spreading is that output will always be real; implying that only the real portion of the transmitter needs to be implemented. Time-domain spreading is obtained by 
transmitting the OFDM symbol followed a permutation of that OFDM symbol.

\section{DESIGN CONSIDERATIONS}

In this section, some of the design considerations, including the frequency synthesizer architecture and the length of the zero-padded prefix, for a MB-OFDM system are discussed.

\subsection{Frequency Planning and Synthesizer Design}

The frequency planning shown in Figure 1 was chosen for two specific reasons: to allow sufficient guard band on the lower side of band 1 and the upper side of band 3, which simplifies the design of a pre-select filter; and to simplify the design of the synthesizer and ensure that the system can switch between the center frequencies within a few nanoseconds.

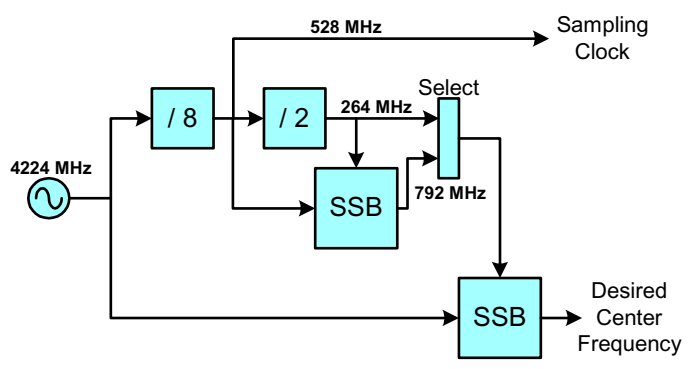

Figure 3 - Example of a MB-OFDM synthesizer architecture.

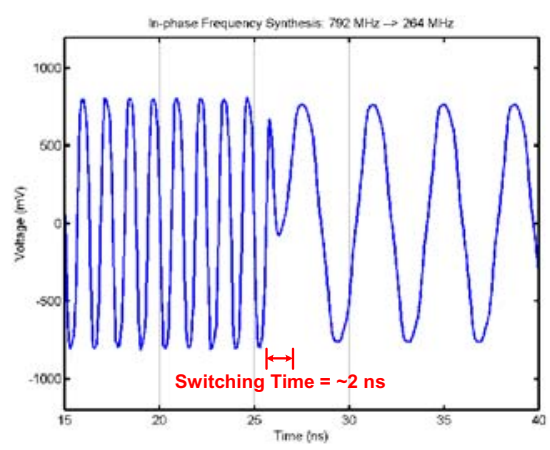

Figure 4 - Circuit-level simulations of the in-phase portion of the frequency-switching architecture

An example of a fast frequency-synthesizer circuit for the MB-OFDM solution is shown in Figure 3. This synthesizer exploits the relationship between the center frequencies for each of the bands and the oscillator frequency. The basic idea is that each of the center frequencies is generated using a single-sideband beat product of the oscillator frequency with another frequency derived from the oscillator. This other frequency is obtained by using a combination of frequency dividers and single-sideband mixers. For example, the center frequency for band 1 is generated by mixing $4224 \mathrm{MHz}$ with $792 \mathrm{MHz}$ to obtain a frequency $3432 \mathrm{MHz}$. The $792 \mathrm{MHz}$ signal is generated by mixing $528 \mathrm{MHz}(=4224$ / 8) and $264 \mathrm{MHz}(=4224$ / 16). Similarly, the center frequencies for bands 2 and 3 can be obtained by mixing $4224 \mathrm{MHz}$ with $264 \mathrm{MHz}$ (= $4224 \mathrm{MHz} / 16)$.

The advantage of this architecture is that all of the center frequencies are generated from a single PLL. Since these frequencies are available at all times, switching between the different sub-bands can be accomplished within a few nanoseconds. The exact time will be limited by the response time of the multiplexer. Figure 4 depicts the plot from a circuit-level simulation of the in-phase portion of frequency-switching architecture shown in Figure 3. From this plot, the nominal switching time is around $2 \mathrm{~ns}$. The result is similar for the quadrature portion of the frequency-switching architecture.

\subsection{Zero-padded Prefix}

An OFDM system offers inherent robustness to multi-path dispersion with a low-complexity receiver. This property is a result of adding a cyclic prefix $(\mathrm{CP})$. A receiver using a CP transforms the linear convolution with the channel impulse response into a circular convolution. Note that a circular convolution in the time domain is equivalent to multiplication in the Discrete Fourier Transform (DFT) domain. Hence, a single-tap frequency domain equalizer is sufficient to undo the effects of the multi-path channel on the transmitted signal.

The length of the CP determines the amount of multi-path energy captured. Multi-path energy not captured during the CP window results in inter-carrier-interference (ICI). Therefore, the CP length needs to be chosen to minimize the impact due to ICI and maximize the multi-path energy collected, while keeping the overhead due to the CP small.

The UWB channel models are highly dispersive; a 4-10 $\mathrm{m}$, non-line-of-sight (NLOS) channel environment has an RMS delay spread of 14 ns [5]. The average captured energy for this channel environment and ICI are illustrated as a function of the CP length in [4]. To capture sufficient multi-path energy and minimize the impact of ICI for all channel environments, the CP length is chosen as $60.6 \mathrm{~ns}$.

OFDM systems typically use a CP to provide robustness against multi-path. However, the same robustness can be obtained by using a zero-padded prefix (ZPP) instead of the CP [6]. The only modification required at the receiver is to collect additional samples corresponding to the 
length of the prefix and to use an overlap-and-add method to obtain the circular convolution property.

(a)

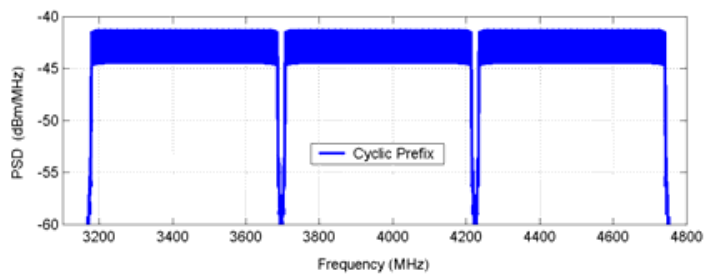

(b)

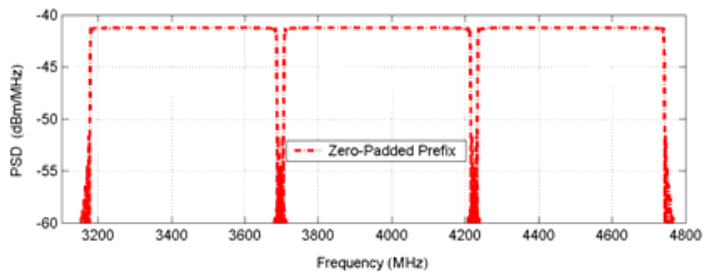

probability of $90 \%$ for a PER of $8 \%$. The link success probability is defined as the percentage of channel realizations for which the system can successfully acquire and demodulate a packet with a PER of less than $8 \%$. These results demonstrate that the MB-OFDM system can attain a distance of approximately 11 meters in all channel environments for a data rate of $110 \mathrm{Mbps}$.

Table $2-90 \%$ link success probability distance for a MBOFDM system.

\begin{tabular}{|c|c|c|c|}
\hline Data Rate & $110 \mathrm{Mb} / \mathrm{s}$ & $200 \mathrm{Mb} / \mathrm{s}$ & $480 \mathrm{Mb} / \mathrm{s}$ \\
\hline AWGN & $20.5 \mathrm{~m}$ & $14.1 \mathrm{~m}$ & $8.9 \mathrm{~m}$ \\
\hline LOS: $0-4 \mathrm{~m}$ & $11.4 \mathrm{~m}$ & $6.9 \mathrm{~m}$ & $2.9 \mathrm{~m}$ \\
\hline NLOS: $0-4 \mathrm{~m}$ & $10.7 \mathrm{~m}$ & $6.3 \mathrm{~m}$ & $2.6 \mathrm{~m}$ \\
\hline NLOS: $4-10 \mathrm{~m}$ & $11.5 \mathrm{~m}$ & $6.8 \mathrm{~m}$ & - \\
\hline
\end{tabular}

\section{SUMMARY}

In this paper, a MB-OFDM system for wireless UWB communication was described. In addition, several design considerations were discussed. Performance results show that this system is able to support a data rate of $110 \mathrm{Mbps}$ at distances of over $10 \mathrm{~m}$.

\section{ACKNOWLEDGEMENTS}

The authors would like to thank Ranjit Gharpurey for helping to develop the MB-OFDM proposal and for generating the frequency synthesis circuit simulations.

\section{REFERENCES}

[1] M. Z. Win and R. A. Scholtz, "On the Robustness of Ultrawide Band Signals in Dense Multipath Environments," IEEE Comm. Letters, Vol. 2, No. 2, February 1998.

[2] V. S. Somayazulu et al., "Design Challenges for Very High Data Rate UWB Systems," Proc. Asilomar Conf. On Systems, Signals and Comp., November 2002.

[3] A. Batra et al., "Multi-band OFDM Physical Layer Proposal for IEEE 802.15 Task Group 3a," IEEE P802.15-03/268r1$T G 3 a$, September 2003.

[4] J. Balakrishnan et al., "A Multi-band OFDM System for UWB Communication," Proc. IEEE Conf. on Ultra Wideband Systems and Technologies, Reston, VA, November 2003.

[5] J. R. Foerster, et al., "Channel Modeling Sub-committee Report Final," IEEE P802.15-02/490r1-SG3a, November 2002.

[6] B. Muquet et al., "Cyclic Prefix or Zero Padding for Wireless Multicarrier Transmission?," IEEE Transactions on Communications, vol. 50, no. 12, December 2002.
Table 2 shows the range, as a function of data rate, for which the MB-OFDM system can achieve a link success 